\title{
First Observations of Cosmic Ray Neutrons by a Mini Neutron Monitor at Riyadh, Saudi Arabia
}

\author{
Maghrabi Abdullrahman, Aldosari Abdulah, Altilasi Mohammed \\ National Center of Nano Technology, King Abdulaziz City for Science and Technology, Riyadh, Saudi Arabia \\ Email: amaghrabi@kacst.edu.sa
}

How to cite this paper: Abdullrahman, M., Abdulah, A. and Mohammed, A. (2020) First Observations of Cosmic Ray Neutrons by a Mini Neutron Monitor at Riyadh, Saudi Arabia. International Journal of Astronomy and Astrophysics, 10, 319-333.

https://doi.org/10.4236/ijaa.2020.104017

Received: October 20, 2020

Accepted: December 8, 2020

Published: December 11, 2020

Copyright $\odot 2020$ by author(s) and Scientific Research Publishing Inc. This work is licensed under the Creative Commons Attribution International License (CC BY 4.0).

http://creativecommons.org/licenses/by/4.0/

\begin{abstract}
In April 2017, a mini neutron monitor (NM) was installed at King Abdulaziz City for Science and Technology (KACST) central Saudi Arabia (Riyadh; cut-off rigidity, $\mathrm{Rc}=14.4 \mathrm{Gv}$ ) for continuous observation of the cosmic ray (CR) neutrons. The detector was built as a major aspect of the international scientific joint effort between the Centre of Space Research (North-West University, Potchefstroom, South Africa) and KACST. The recorded data correspond to low energy neutrons that primarily have energies lower than 20 $\mathrm{GeV}$. In this paper, a brief description about the mini NM detector will be given. The influence of atmospheric pressure on the recorded CR neutrons was studied and the barometric coefficient was calculated and used to eliminate the pressure effects from the measured data. The obtained coefficient was consistent with those previously obtained by several investigators. The daily variation of the CR neutron was studied and characterized. Short-term CR periodicities, such as the 27-day period, and its two harmonics, were identified. The obtained periodicities are in agreement with those reported by different researchers. The obtained results from this detector have been compared to the existing $1 \mathrm{~m}^{2}$ scintillator detector showing comparable results. Long-term data from this detector will be of incredible significance to the research community to investigate several types of $\mathrm{CR}$ variations resulting from solar activity at such high cut off rigidity site.
\end{abstract}

\section{Keywords}

Mini Neutron Monitor, Cosmic Ray, Saudi Arabia, Periodicities

\section{Introduction}

Cosmic rays (CRs) are energetic charged particles originating from deep spaces that reach the top of our atmosphere about $30 \mathrm{~km}$ above the Earth's surface. 
They come from a variety of sources including our own Sun, stars and distant objects such as black holes and active galaxies. Most CRs are the nuclei of atoms, ranging from the lightest to the heaviest elements in the periodic table, as well as, high energy electrons, positrons, and other subatomic particles. Cosmic ray studies are linked to many branches of physics and astrophysics [1] [2]. Cosmic ray experiments allow high-energy physics researchers to extend their interaction models to super-accelerator energies, leading to the discovery of many elementary particles [3] [4]. Cosmic ray observations provide information for astrophysicists about the interstellar medium, magnetic fields and the nature of their sources [5] [6] [7]. Cosmic rays studies have been found to be a valuable tool to search for large disturbed phenomena in the heliosphere [2] [8]. Additionally, recent studies have shown that CRs, due to their capacity to cause ionization, influence various physio-chemical processes, which in turn affect the global weather and climate [9] [10] [11] [12].

Solar disturbances change the energy output from the sun, which affects the interplanetary medium and the terrestrial environment [13]. These variations affect the propagation of the high-energy $\mathrm{CR}$ in the heliosphere, and their rate at the top of the atmosphere is modulated [14] [15].

Knowledge of CR flux is important to understand the effect of the solar activity and CR on the physical and chemical properties of the Earth's atmosphere, e.g. [9].

Since their discovery by Viktor Hess in 1912, different types of space-based and ground-level CR detectors have been developed to study the CR modulations in different time scales [16] [17]. These detectors were sensitive to various components of CRs.

Because of its high cut off rigidity and unique location [18] [19] [20] [21], monitoring CR variations in Saudi Arabia is of an incredible significance for the research community. CR monitoring program began in Saudi Arabia in 2002 by installing a single channel detector for CR muon observations [22]. In 2017, as a part of our radiation detector laboratory activities [22], we have installed a mini-neutron monitor in Riyadh, Saudi Arabia $(\mathrm{Rc}=14.4 \mathrm{GV})$, aiming to monitor and explore the variations of CR neutrons at various time scales. This detector was designed and built by the Centre of Space Research (North-West University, Potchefstroom, South Africa) and has been installed in a few locations around the world and demonstrates its usefulness with accuracy as comparable as the standard NM [23]-[29].

The paper is organized as follows: brief description about this detector will be given in Section 2. The obtained results will be presented and discussed in Section 3 , whereas the conclusions and future work will be summarized in the last section.

\section{The Detection System}

\subsection{Concept of Neutron Monitors}

Neutron monitors are the standard (and most commonly use) ground-based in- 
strument and most effective tool for monitoring the variations of the low energy (500 Mev to $20 \mathrm{GeV}$ ) part of the CR spectrum [30].

A standard neutron monitor, as developed by Simpson and his colleagues in 1953 [31], is designed as a counter surrounded with consecutive layers of a light-element material and lead (Figure 1).

The outer layer reflector shields the detector from external thermal neutrons and is transparent to cascade neutrons, which are multiplied in the lead producer (producing additional low energy neutrons via nuclear interactions to increase the monitor's detection efficiency). The inner moderator slows nuclei down in order to enhance their registration probability. The multiplied and decelerated neutrons are then counted via electrical pulses produced in a proportional counter tube, traditionally filled with either $\mathrm{BF} 3$ or $3 \mathrm{He}$ gas [32].

The design of the basic NM has changed slightly over the years [17] [19] [33] [34]. Several NM monitors have been developed with different number of tubes and different configurations to optimize the counting rate capabilities. These include the development of the second generation of super monitors [17] [33] [35].

In 2001, the idea of developing small sizes of the standard NM was introduced by [23] to inter-calibrate the existed NM worldwide. The aim of this design was to develop portable easy to move and cost effective detector for CR neutron variations which can be operated in, even, remote locations [16] [36].

After several years of operations in different places, these types of detectors have shown excellent performance and comparable data with the standard NMs, which are then named mini NMs [37]. Since their first development, the mini-NMs design has been constantly optimized and the counting electronics updated. The detector used in this work is the up to dated version of the developed mini NM [36].

\subsection{KACST Mini Neutron Monitor}

Brief description of the installed detector will be presented below and detailed

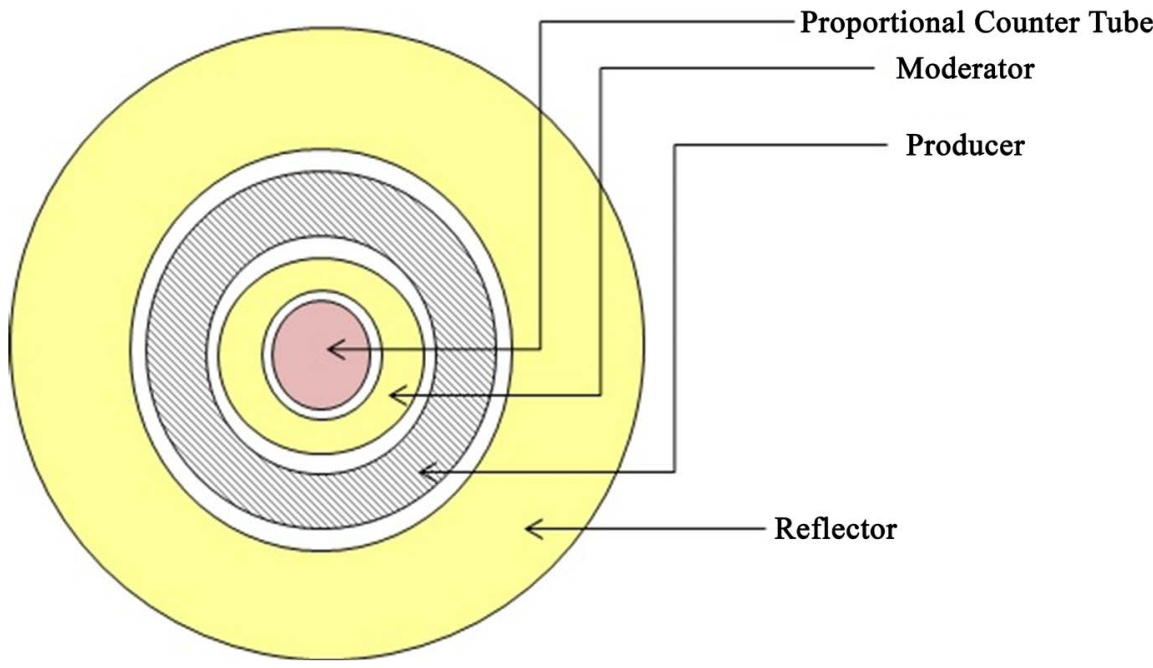

Figure 1. Schematic diagram shows the structure of the standard neutron monitor. 
information about the detector, mechanical design, electronic components, and calibrations procedures can be found in several publications [23] [26] [36] [37] [38] [39].

The detector (Figure 2) has a dimension of $85 \mathrm{~cm}$ long and $60 \mathrm{~cm}$ of radius which is around $1 / 3$ of the original size of the typical full size NM64 neutron monitor. However, the count rate of this monitor is similar to a standard NM64 detector [36]. The utilized counter is LND2043 type and filled by BF3 gas with a pressure of $933 \mathrm{hPa}$. The pressure in the standard NM64 detectors is 300 mbar. The high pressure used in the mini NM compensates for the reduced efficiency of the mini-NM as compared to the much larger full-size NM64.

The detector comprises of $63 \mathrm{~cm}$ long counter encompassed by a $2 \mathrm{~cm}$ polyethylene moderator which was surrounded by a $5 \mathrm{~cm}$ neutron producer made of lead. The lead ring is surrounded by a $9.5 \mathrm{~cm}$ polyethylene reflector. High voltage unit incorporated with the low-noise pre-amplifier was utilized to generate the high voltage ( $2300 \mathrm{~V}$ for the BF3 detector) for the detector tube. Atmospheric pressure was measured by Vaisala pressure transducer.

The detector has a GPS module for location determination. A PIC32 microcontroller was implemented to control the entire activity of the detector. A data acquisition unit was developed for logging the data, interfacing with the electronics, and storing the data. The electronics make data records with 1-second resolution.

The detector is situated in the radiation detector laboratory at KACST and has been in operation since April 2017. During this period, the detector went through periods of downtime due to technical issues which caused some periods of missing data. Thus, data for the period between April 2017-January 2018 were considered for the purpose of this study.

Also, cosmic ray data acquired from KACST muon detector installed at a similar site were utilized for correlations with those obtained from the mini neutron monitor for a similar timeframe. The muon detector is a single channel $1 \mathrm{~m}^{2}$ plastic scintillator detector detects high energy CR muons. Details regarding this detector are given in several research articles [11] [40].

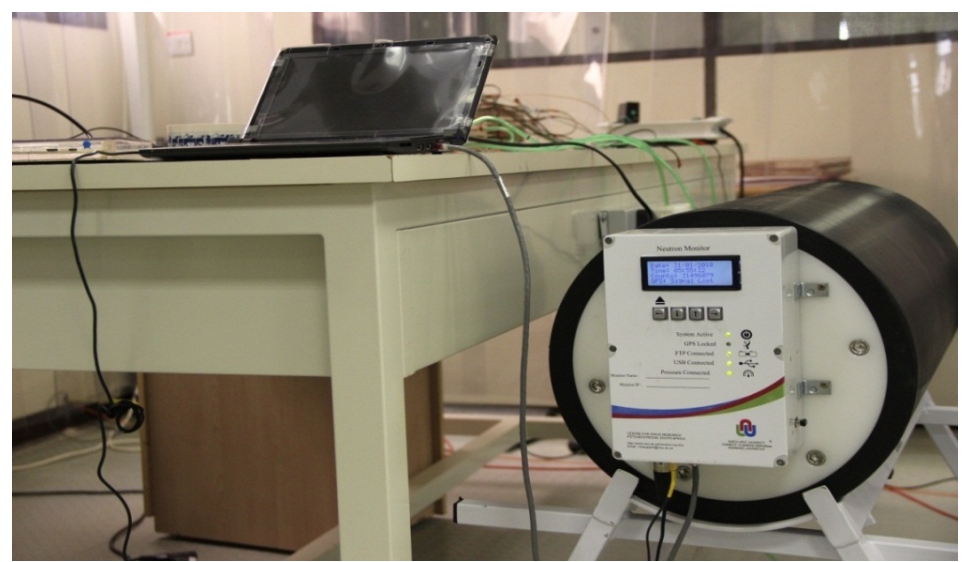

Figure 2. The mini neutron monitor installed at KACST station. 


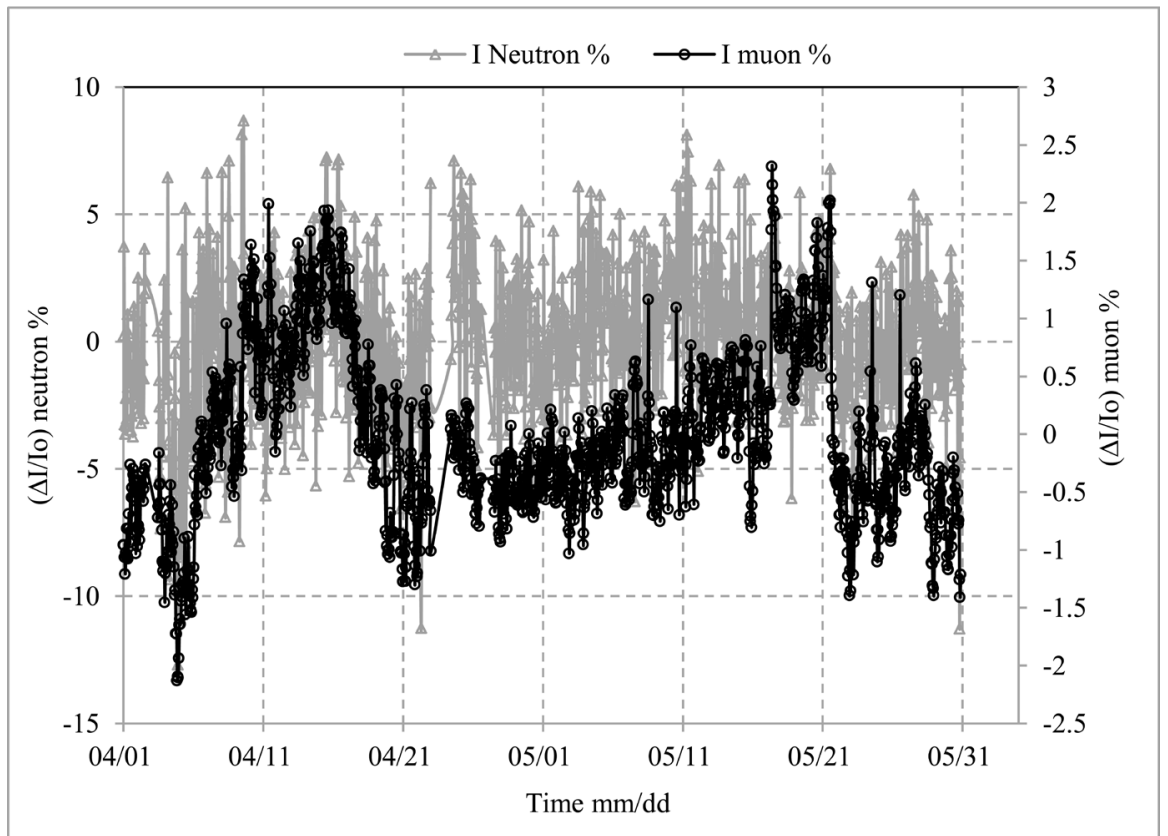

Figure 3. Hourly time series of the relative raw cosmic ray intensities $(\Delta \mathrm{I} / \mathrm{Io})$ measured by the mini neutron monitor and KACST single channel muon detector for the period April to May 2017.

Figure 3 demonstrates the relative count rates of the mini NM for the time of two month between April to May 2017 compared with those from the $1 \mathrm{~m}^{2}$ muon detector. It can be seen that the behaviour of the mini NM is practically like those of the muon detector for most of the period.

\section{Results and Discussions}

\subsection{Pressure Corrections}

To properly consider the impact of the solar activities on the primary CR, atmospheric effects on the secondary CR must be evaluated. Atmospheric pressure is the most effective atmospheric variable influencing the flux of the secondary CR particles (e.g., Dorman 2004). Figure 4 is an example indicating the hourly time series of the atmospheric pressure variations with (a) cosmic ray neutrons and (b) cosmic ray muons; for a time of around one month. The atmospheric pressure determines the atmospheric mass that the CR particles need to go through. In this manner, an expansion in the atmospheric pressure will reduce the measure of the detected particles and vice versa. The diurnal variations of the atmospheric pressure causes rising and/or potentially lowering down the generation layer that at that point influences the CR flux.

The pressure effect on the secondary cosmic ray component can be expressed as:

$$
\frac{I-I_{0}}{I}=\alpha\left(P-P_{0}\right)
$$

$I$, is the cosmic ray rate at pressure $P, I_{0}$ is the rate at the mean atmospheric 

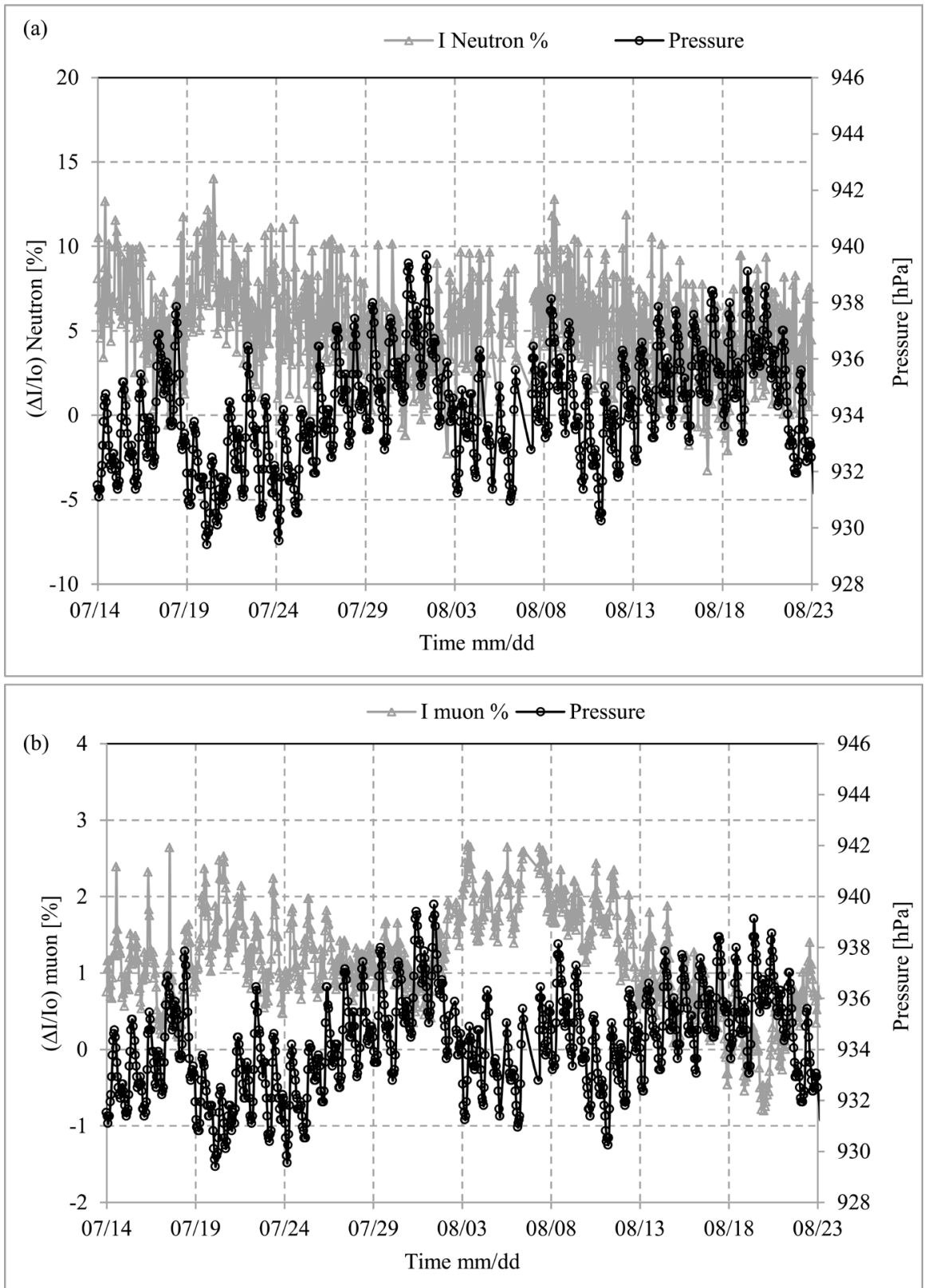

Figure 4. Hourly variations of the atmospheric pressure and cosmic ray relative intensities $(\Delta \mathrm{I} / \mathrm{Io})$ from (a) the mini neutron monitor and (b) KACST muon detector; for the period between July - August 2017.

pressure at the site altitude (here $940 \mathrm{mbar}$ ), and $\alpha$ is the barometric coefficient. $\alpha$ can be determined experimentally by using a simple linear regression between the atmospheric pressure and the secondary cosmic ray rate.

Figure 5 indicates the hourly values of the atmospheric pressure plotted against the cosmic ray relative intensities from (a) the mini neutron monitor and (b) KACST muon detector; during the study period. It is evident that secondary cosmic rays decrease as the atmospheric masses above the detector increases.

Regression analyses between the atmospheric pressure and CR neutrons yields a barometric coefficient of $0.61 \% / \mathrm{mbar} \pm 0.01 \% / \mathrm{mbar}$ with a 0.65 correlation 

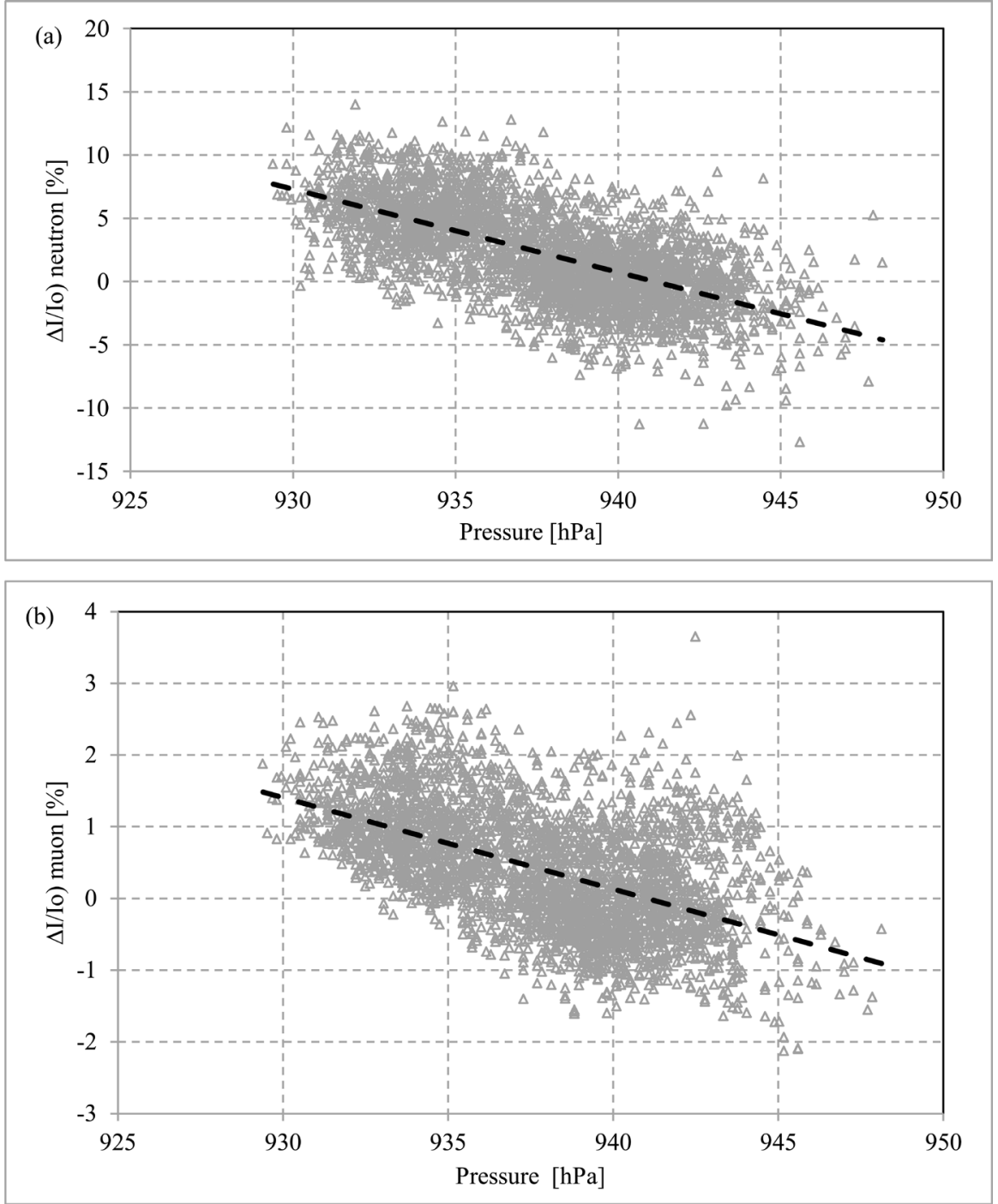

Figure 5. Scatter plot of the hourly values of atmospheric pressure and cosmic ray relative intensities $(\Delta \mathrm{I} / \mathrm{Io})$ recorded by (a) the mini neutron and (b) KACST muon detector during the study period. The straight dashed line is the best linear fit to the data here the slope is the barometric coefficient found in Equation (1).

coefficient $(r)$. The obtained barometric coefficient for the neutron monitor is near those obtained by several investigators [41] [42]. The barometric coefficient for the muon detector was $0.067 \% / \mathrm{mbar} \pm 0.003 \% / \mathrm{mbar}$ with a correlation coefficient of 0.56 . This coefficient was, in any case, small and somewhat not quite the same as those detailed by different researchers. This might be because of the dependence of these coefficients on components, for example, the size and the arrangement of the muon detector just as the energy of the recorded particles. For instance, De Mendonca et al., [43] used data from the Global Muon Detector Network (GMDN) to study the atmospheric impacts on the cosmic ray muons and found that the barometric coefficient values range between $0.114 \% / \mathrm{mbar}$ and $0.168 \% / \mathrm{mbar}$.

The obtained coefficients, from the neutron and muon data, were utilized to 
eliminate the effect of the atmospheric pressure from the collected data using Equation (1). When the correction for local pressure effect has been made, result variations in $\mathrm{CR}$ rate reflect the condition of the heliosphere and the solar wind, which modulates the intensity of the primary CR flux.

Figure 6 demonstrates the consistencies between the behaviour of the pressure corrected CR counts from the mini NM and the muon detector for a period of two months.

Figure 7 is a scatter plot of the daily pressure-corrected relative muon intensities against those measured by the mini NM for the considered period. The regression equation between the two values was:

$$
\frac{\Delta I}{I}(N M)=4.63 \frac{\Delta I}{I}\left(1 \mathrm{~m}^{2}\right)+0.41
$$

The results from the above two figures demonstrate the good detectability and comparability of CRs recorded by the two detectors utilizing different techniques. However, the deviations of the data from the 1:1 line can be attributed to a few technical factors, for example, the differences in geometry, efficiency, and response functions of the two detectors. Additionally, the effect of atmospheric conditions on the CR muons is another factor affects the count rate of the $\mathrm{CR}$ muons.

\subsection{Diurnal Variations}

The daily variations of the CR are because of the daily rotation of the earth around the sun, which results recording CR with greater and lesser intensities.

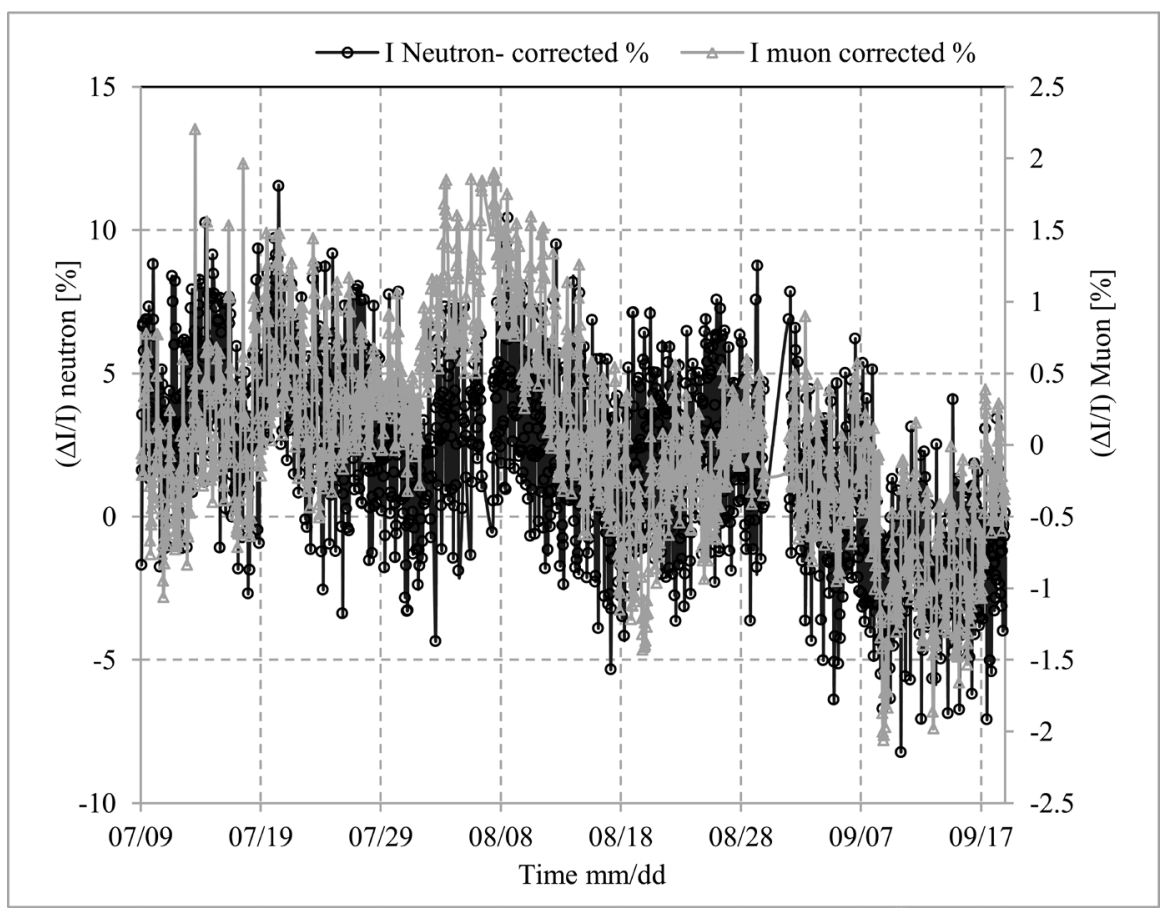

Figure 6. Pressure corrected relative intensities ( $\Delta \mathrm{I} / \mathrm{Io})$ from the mini NM and muon detector for the period July-September 2017. 


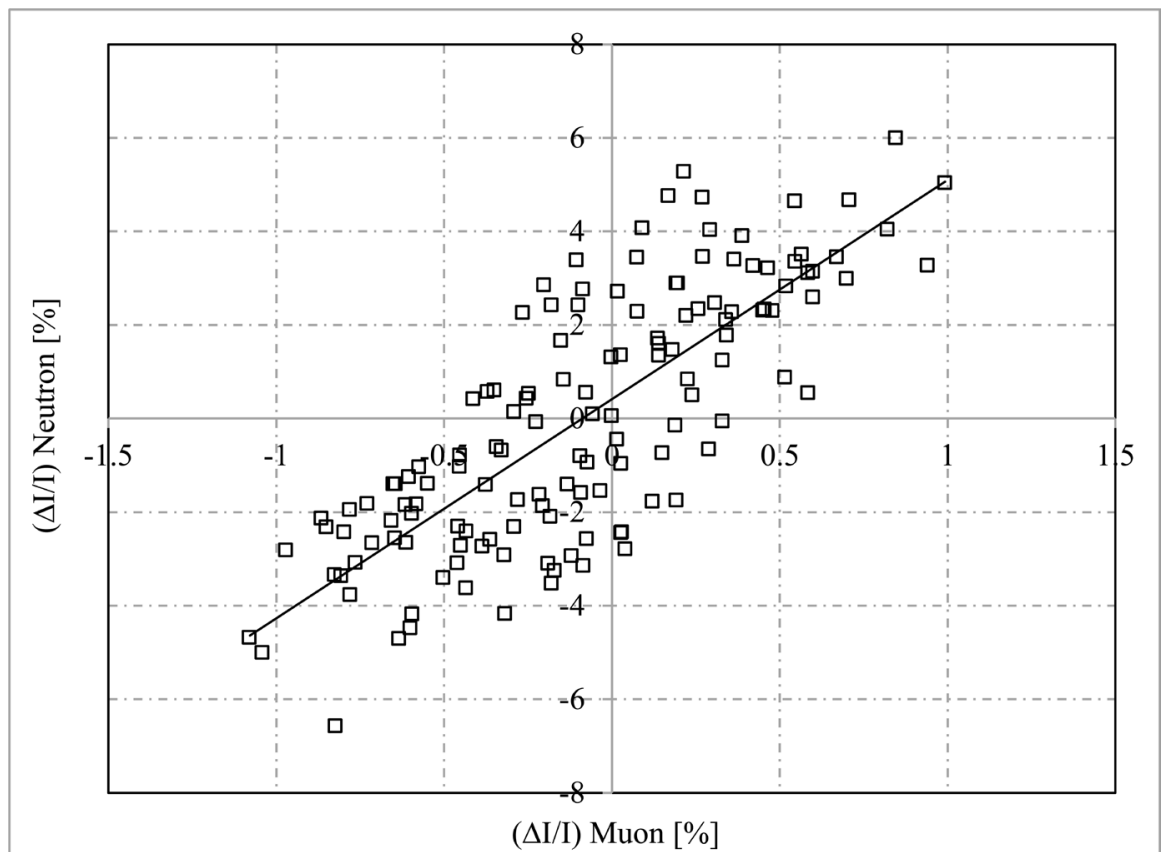

Figure 7. Comparisons between the daily mean values of the muon relative intensities recorded by the $1 \mathrm{~m}^{2}$ KACST detector and neutron relative intensities observed by the mini neutron monitor. The solid line is the regression fit between the two variables.

Characterizing the diurnal variations of the CR is important to understand the physical properties of the heliosphere and the Earth's magnetosphere. Diurnal variations can be characterized by the amplitude (maximal value) and phase (time of the maximal amplitude) [44] [45].

Figure 8 indicates daily variations of the cosmic ray (a) muons as observed by the KACST muon detector and (b) neutrons recorded by the mini NM during the study period. It is clearly observed that the daily variations of the CR neutrons, throughout the twenty-four hours are higher (about 1.24\%) than that of the CR muon (0.63\%).

The daily variations of the muon data described by a gradual increase in rate from 3:00 local time (LT) to achieve the maximum around 13:00 $\mathrm{h} \mathrm{LT}$. Then the maximum is maintained until 16:00 h LT. After that, it decreases again to achieve its minimum. On the other hand, the NM rates increased from 5:00 h LT till it achieves the maximum around 14:00 $\mathrm{h} \mathrm{LT}$ and stayed around the maximum values $(0.8 \%)$ until $16: 00 \mathrm{~h} \mathrm{LT}$ when it declines significantly to reach $(-0.3)$ at 19:00 h LT. At that point, it increases again to achieve an estimation of 0.12 around 22:00 h LT, while it at that point, decreases to its minimum value. Clearly, maximum intensities (phase) from the two detectors occurred at 16:00 h LT corresponding to 13:00 h UT. These results are consistent with those obtained previously [44] [45] [46].

\subsection{Short Term Periodicities}

Power spectral investigations have been carried out using Fourier transform 

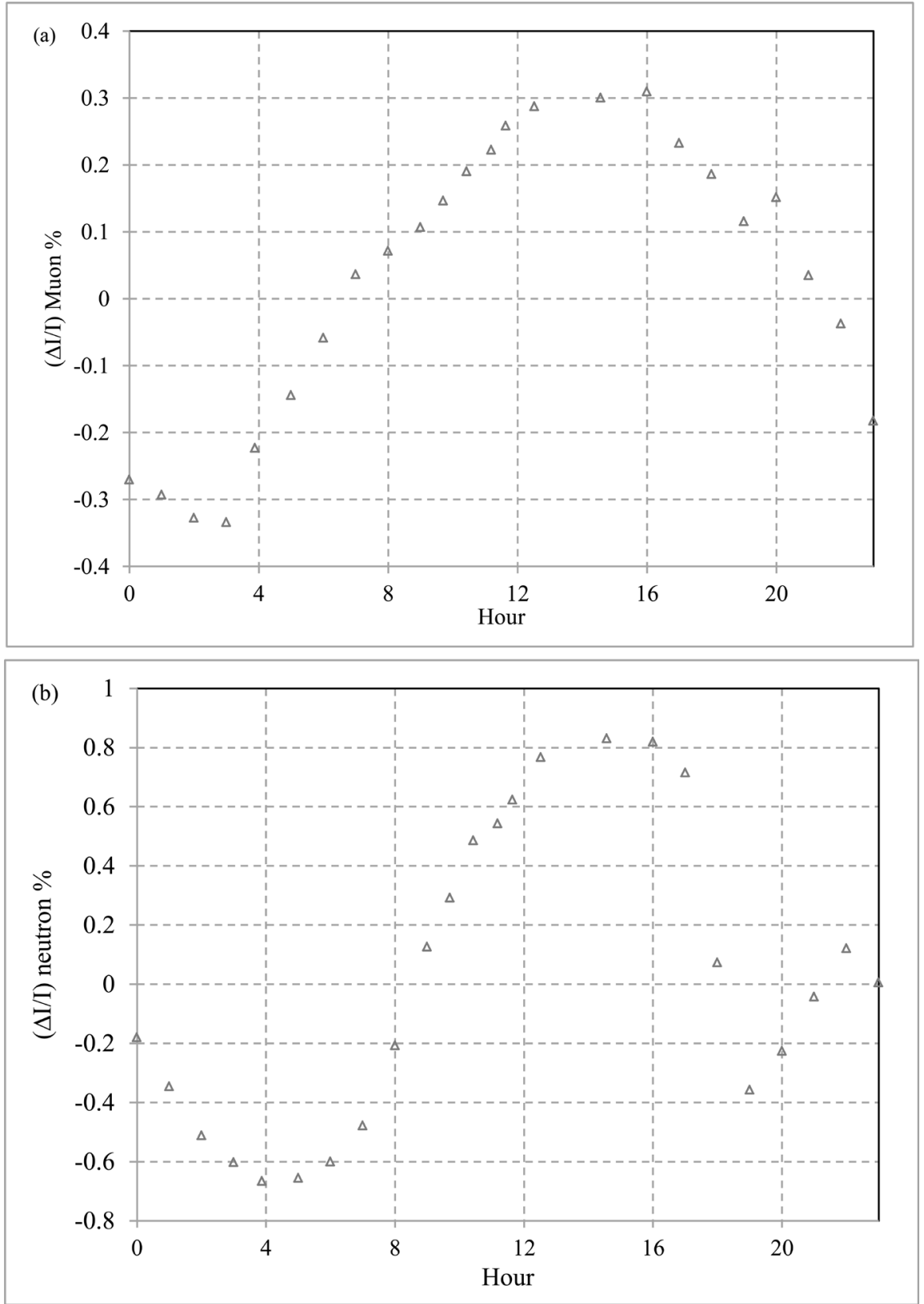

Figure 8. Diurnal variation of the pressure corrected cosmic ray relative intensities $(\Delta \mathrm{I} / \mathrm{Io})$ recorded by (a) the muon detector and (b) the mini NM during the study period.

technique to investigate short term periodicities in the CR data gathered by the mini NM and muon detectors during the study period. Figure 9 is a power spectral density demonstrating the significant peak of different amplitudes and strengths over every one of the frequencies for the (a) neutron monitor and (b) muon detector.

The strength and the amplitudes of the peaks are diverse for the two detectors and generally found in the range run between $0-0.05 \mathrm{l} / \mathrm{h}$. The neutron monitor has a few significant peaks (higher than $99 \%$ significant level were considered), for example, the 15.2 days, 12.3 days, 7.4 days, 5.6 days, and 3.4 days. On the 

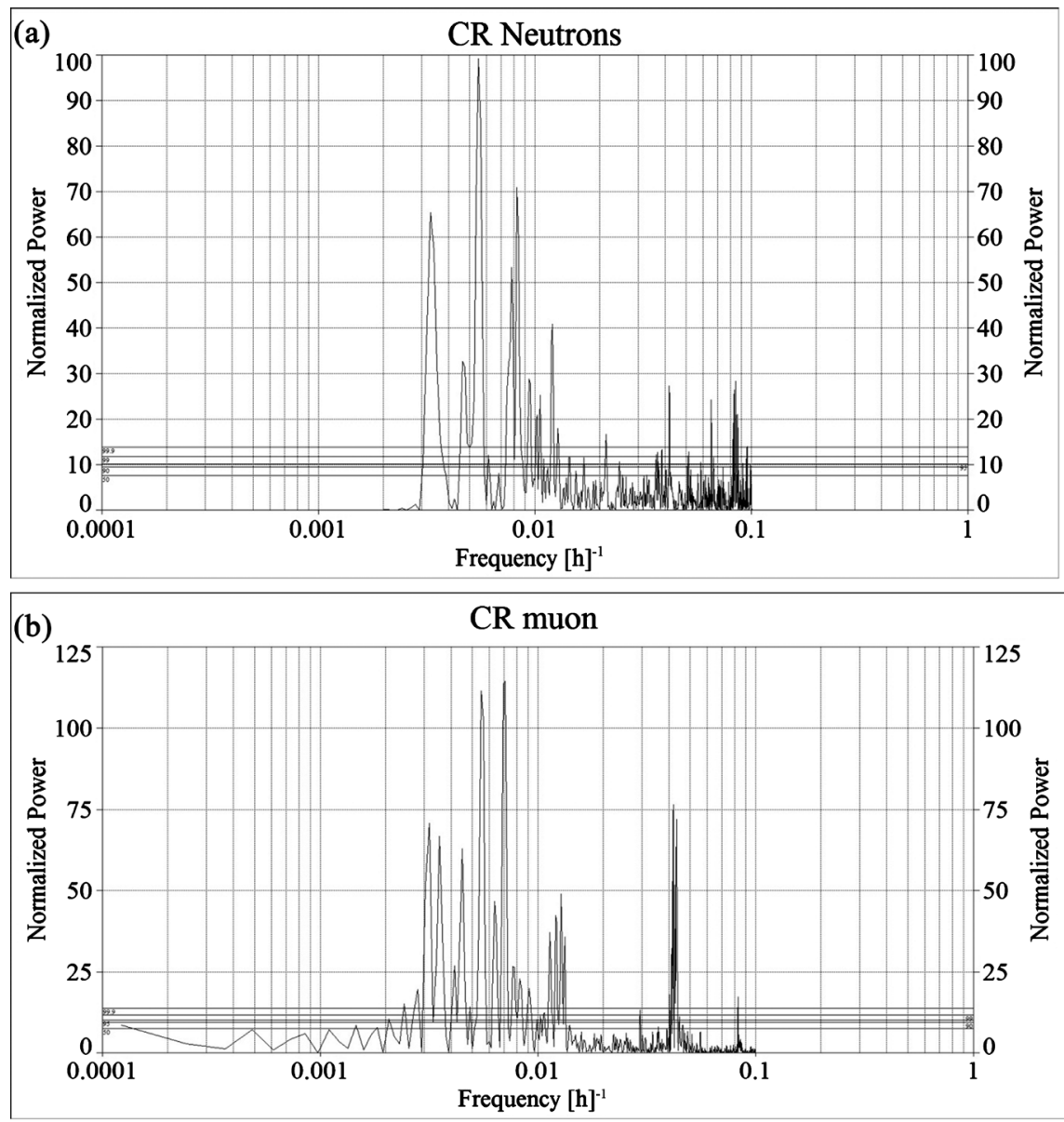

Figure 9. Power spectral density for the pressure corrected cosmic rays recorded by (a) the mini neutron monitor and (b) KACST muons detector for the considered period of study. The frequency unit is (1/hour). The horizontal lines are $50 \%, 90 \%, 95 \%, 99 \%$, and $99.9 \%$ confidence levels.

other hand, the muon data demonstrates the 11.7 days, 7.4 days, 5.8 days, and 3.4 days. On the frequency range between 0.04 and 0.045 , the 24 hours periodicity is obvious in the spectra of the two data, in which it was clearer for the muon data than that of the neutron monitor. These obtained periodicities are in agreements with those found by several investigators [47] [48] [49] [50] [51].

\section{Conclusions}

Solar disturbances affect the propagation of the primary high-energy cosmic rays in the heliosphere, and their rate at the top of the atmosphere is modulated. Cosmic ray neutrons represent the low energy (below $20 \mathrm{GeV}$ ) part of the extensive air shower produced in the atmosphere by the interactions between the primary CRs and atmospheric molecules. Neutron monitors are the standard and most commonly use ground-based instrument for monitoring and studying the variations of $\mathrm{CR}$ neutrons at various time scales.

In mid-2017, a mini-neutron monitor was installed at (KACST) central Saudi Arabia (Riyadh; Rc $=14.4 \mathrm{Gv}$ ) to supplement the presently existing $\mathrm{CR}$ detectors 
(scintillator detector, CARPET detector, and multi-wires detector).

The effect of the atmospheric pressure on the mini neutron monitor data was studied and the required coefficient was determined. It was found that the atmospheric pressure is anti-correlated with the CR data. The barometric coefficient for this analysis was $0.61 \% / \mathrm{mbar} \pm 0.01 \% / \mathrm{mbar}$ with a 0.65 correlation coefficient and was close to those reported by several investigators. The detectability of the mini neutron monitor was tested against the $1 \mathrm{~m}^{2}$ muon detectors and showed comparable behaviours. These include the daily variations of the CR particles which presented diurnal behaviour similar to those previously reported.

Fourier transform analyses were carried out to the collected data to investigate the short term periodicities exhibited by CR particles. Some short-term periodicities, for example, the 11 - 12 days, 7 days, 5 - 6 days, and 4.3 days were identified. The obtained periodicities are similar to those reported by different researchers.

Despite the fact that the installed mini NM has a small size in comparisons with the standard neutron monitors, it indicated high abilities to record low-energy cosmic rays and a strong response of their variability.

With its unique position in this part of the world, long and short term data from this detector can be utilized to supplement CR data from neutron monitor and muon detector observations at different places around the world to explore the cosmic ray variations beyond the energy ranges of extant cosmic ray detectors.

\section{Acknowledgements}

The authors would like to thank King Abdulaziz City for Science and Technology (KACST) for supporting this work. Also, we would like to thank the anonymous reviewers for their valuable comments and recommendations.

\section{Conflicts of Interest}

The authors declare no conflicts of interest regarding the publication of this paper.

\section{References}

[1] Dobrotin, N., Zatsepin, G., Nikolsky, S. and Slavatinsky, S. (1985) Beginning of High-Energy Physics in Cosmic Rays. In: Sekido, Y. and Elliot, H., Eds., Early History of Cosmic Ray Studies. Personal Reminiscences with Old Photographs, Astrophysics and Space Science Library, Vol. 118, Springer, Berlin, 225. https://doi.org/10.1007/978-94-009-5434-2_23

[2] Dorman, L. (2004) Cosmic Rays in the Earth's Atmosphere and Underground. Kluwer Academic Publishers, Dordrecht. https://doi.org/10.1007/978-1-4020-2113-8

[3] Brown, L. and Hoddeson, L. (1983) The Birth of Particle Physics. In: International Symposium on the History of Particle Physics, Cambridge University Press, Cambridge.

[4] Blandford, R. and Eichler, D. (1987) Particle Acceleration at Astrophysical Shocks: A Theory of Cosmic Ray Origin. Physics Reports, 154, 1-75. https://doi.org/10.1016/0370-1573(87)90134-7 
[5] Duvernois, M. (1999) Topics in Cosmic-Ray Astrophysics, Horizons in World Physics. Vol. 230, Nova Science, Commack.

[6] Cirkel-Bartelt, V. (2008) History of Astroparticle Physics and Its Components. Living Reviews in Relativity, 11, Article No. 2. https://doi.org/10.12942/lrr-2008-2

[7] Abraham, J., Abreu, P., Aglietta, M., Aguirre, C., et al. (2008) Correlation of the Highest Energy Cosmic Rays with the Positions of Nearby Active Galactic Nuclei. Astroparticle Physics, 29, 188-204. https://doi.org/10.1016/j.astropartphys.2008.01.002

[8] Kudela, K. and Storini, M. (2009) Possible Tools for Space Weather Issues Form Cosmic Ray Continuous Records. Advances in Space Research, 37, 1443-1449. https://doi.org/10.1016/j.asr.2006.02.058

[9] Haigh, J. (1996) The Impact of Solar Variability on Climate. Science, 272, 981-984. https://doi.org/10.1126/science.272.5264.981

[10] Marsh, N. and Svensmark, H. (2003) Solar Influence on Earth's Climate. Space Science Reviews, 107, 317-325. https://doi.org/10.1023/A:1025573117134

[11] Maghrabi, A. (2019) Multi-Decadal Variations and Periodicities of the Precipitable Water Vapour (PWV) and Their Possible Association with Solar Activity: Arabian Peninsula. Journal Solar Terrestrial Physics, 185, 22-28. https://doi.org/10.1016/j.jastp.2019.01.011

[12] Maghrabi, A. and Kudela, K. (2019) Relationship between Time Series Cosmic Ray Data and Aerosol Optical Properties: 1999-2015. Journal Solar Terrestrial Physics, 190, 36-44. https://doi.org/10.1016/j.jastp.2019.04.014

[13] Zhao, J., et al. (2004) The Effect of Solar Activity on the Annual Precipitation in the Beijing Area. Chinese Journal of Astronomy and Astrophysics, 4, 189-197. https://doi.org/10.1088/1009-9271/4/2/189

[14] Xanthakis, J., Mavromichalaki, H. and Petropoulos, B. (1989) Time Evolution of Cosmic Ray Intensity Modulation. Solar Physics, 122, 345-363. https://doi.org/10.1007/BF00913001

[15] Cane, H., Wibberenz, G., Richardson, I. and Rosenvinge, T. (1999) Cosmic Ray Modulation and the Solar Magnetic Field. Geophysical Research Letters, 26, 565-568. https://doi.org/10.1029/1999GL900032

[16] Butikofer, R. (2018) Ground-Based Measurements of Energetic Particles by Neutron Monitors. In: Solar Particle Radiation Storms Forecasting and Analysis, the Hesperia Horizon 2020 Project and Beyond, Springer Nature, Cham, 95-112. https://doi.org/10.1007/978-3-319-60051-2_6

[17] Stoker, P. (2009) The IGY and Beyond: A Brief History of Ground-Based Cosmic-Ray Detectors. Advances in Space Research, 44, 1081-1095. https://doi.org/10.1016/j.asr.2008.10.037

[18] Shea, M. and Smart, D. (1982) Possible Evidence for a Rigidity-Dependent Release of Relativistic Protons from the Solar Corona. Space Science Reviews, 32, 251-271. https://doi.org/10.1007/BF00225188

[19] Bieber, J., Clem, J., Duldig, M., et al. (2004) Latitude Survey Observations of Neutron Monitor Multiplicity. Journal of Geophysical Research, 109, A12106. https://doi.org/10.1029/2004JA010493

[20] Herbst, K., Koppm, A. and Heber, B. (2013) Influence of the Terrestrial Magnetic Field Geometry on the Cutoff Rigidity of Cosmic Ray Particles. Annales Geophysicae, 31, 1637-1643. https://doi.org/10.5194/angeo-31-1637-2013

[21] Lara, A., Borgazzi, A. and Caballero-Lopez, R. (2016) Altitude Survey of the Galac- 
tic Cosmic Ray Flux with a Mini Neutron Monitor. Advances in Space Research, 58, 1441-1451. https://doi.org/10.1016/j.asr.2016.06.021

[22] Maghrabi, A., Al Harbi, H., Al-Mostafa, Z., Kordi, M. and Al Shehri, S. (2012) The KACST Muon Detector and Its Application to Cosmic-Ray Variations Studies. Advances in Space Research, 50, 700-711. https://doi.org/10.1016/j.asr.2011.10.011

[23] Moraal, H., Benadie, A., de Villiers, D., et al. (2001) A Mobile Neutron Monitor to Intercalibrate the Worldwide Network. Proceedings of the 27 th International Cos mic Ray Conference, Hamburg, 7-15 August 2001, Vol. 10, 4083.

[24] Heber, B., Galsdorf, D., Herbst, K., Gieseler, J., et al. (2014) Mini Neutron Monitor Measurements at the Neumayer III Station and on the German Research Vessel Polarstern 2014. Journal of Physics: Conference Series, 632, Article ID: 012057. https://doi.org/10.1088/1742-6596/632/1/012057

[25] Krüger, H. and Moraal, H. (2010) A Calibration Neutron Monitor: Statistical Accuracy and Environmental Sensitivity. Advances in Space Research, 46, 1394-1399. https://doi.org/10.1016/j.asr.2010.07.008

[26] Krüger, H. and Moraal, H. (2013) Neutron Monitor Calibrations: Progress Report. Journal of Physics. Conference Series, 409, Article ID: 012171. https://doi.org/10.1088/1742-6596/409/1/012171

[27] Poluianov, S., Usoskin, I., Mishev, A., et al. (2015) Mini Neutron Monitors at Concordia Research Station, Central Antarctica. Journal of Astronomy and Space Sciences, 32, 281-287. https://doi.org/10.5140/JASS.2015.32.4.281

[28] Medina, J., Blanco, J., Garca, O., et al. (2013) Castilla-La Mancha Neutron Monitor. Nuclear Instruments and Methods in Physics Research Section A, 727, 97-103. https://doi.org/10.1016/j.nima.2013.06.028

[29] Kuwabara, T., Bieber, J., Clem, J., Evenson, P., Pyle, R., et al. (2006) Real-Time Cosmic Ray Monitoring System for Space Weather. Space Weather, 4, 1-10. https://doi.org/10.1029/2005SW000204

[30] Stoker, P., Dorman, L. and Clem, J. (2000) Neutron Monitor Design Improvements. Space Science Reviews, 93, 361-380. https://doi.org/10.1023/A:1026560932107

[31] Simpson, J., Fonger, W. and Treiman, S. (1953) Cosmic Radiation Intensity-Time Variation and Their Origin. I. Neutron Intensity Variation Method and Meteorological Factors. Physical Review, 90, 934-950.

https://doi.org/10.1103/PhysRev.90.934

[32] Simpson, J. (1957) Cosmic-Radiation Neutron Intensity Monitor. Annals of the International Geophysical Year, 4, 351-373. https://doi.org/10.1016/B978-1-4832-1304-0.50020-8

[33] Simpson, J. (2000) The Cosmic Ray Nucleonic Component: The Invention and Scientific Uses of the Neutron Monitor-(Keynote Lecture). Space Science Reviews, 93, 11-32. https://doi.org/10.1023/A:1026567706183

[34] Signoretti, F. and Storini, M. (2011) A New Modular Cosmic-Ray Detector. Astrophysics and Space Science, 7, 11-14. https://doi.org/10.5194/astra-7-11-2011

[35] Usoskin, I., Kovaltsov, G., Kananen, H. and Tanskanen, P. (1997) The World Neutron Monitor Network as a Tool for the Study of Solar Neutrons. Annales Geophysicae, 15, 375-386. https://doi.org/10.1007/s00585-997-0375-9

[36] Toit, S., Poluianov, S., van der, C., et al. (2020) The Mini-Neutron Monitor: A New Approach in Neutron Monitor Design. Journal of Space Weather and Space Climate, 10, 39. https://doi.org/10.1051/swsc/2020038

[37] Krüger, H., Moraal, H., Nel, R., Krüger, H.G. and O'Kennedy, M. (2015) The Mini 
Neutron Monitor Programme. Proceedings of the 34th International Cosmic Ray Conference (ICRC2015), The Hague, 30 July-6 August 2015, 223. https://doi.org/10.22323/1.236.0223

[38] Krüger, H., Moraal, H., Bieber, J.W., et al. (2005) Latitude Surveys with a Calibration Neutron Monitor. 29 th International Cosmic Ray Conference, Tsukuba, Vol. 2, 473-476.

[39] Krüger, H., Moraal, H., Bieber, J., et al. (2003) First Results of a Mobile Neutron Monitor to Intercalibrate the Worldwide Network. Proceedings of the 28 th International Cosmic Ray Conference, Vol. 6, Tsukuba, 31 July-7 August 2003, 3441-3444.

[40] Maghrabi, A., Alghamdi, A., Alotaibi, R., Almutariand, M. and Garawi, M. (2014) On the Calibration of a Single Channel Cosmic Ray Particle Detector. SPIE 9154, High Energy, Optical, and Infrared Detectors for Astronomy, Vol. 6, 91542K. https://doi.org/10.1117/12.2059231

[41] Rogelio, C. and Valdés-Galicia, J. (2000) Variations in Cosmic Radiation Intensity Associated with the Barometric Effect. Geofisica Internacional, 39, 135-137.

[42] Platanos, I., Gerontidou, M., Paschalis, P. and Mavromichalaki, H. (2015) Long Term Variation of the Barometric Coefficient of the Neutron Component of Cosmic Rays. 12th Hellenic Astronomical Society Conference, Thessaloniki, 28 June-2 July 2015, 1-2.

[43] De Mendonca, R., Braga, C., Echer, E., et al. (2016) The Temperature Effect in Secondary Cosmic Ray (Muons) Observed at the Ground: Analysis of the Global Muon Detector Community Data. The Astrophysical Journal, 830, 88. https://doi.org/10.3847/0004-637X/830/2/88

[44] Kudela, K., Firoz, K.A., Langer, R., et al. (2003) On Diurnal Variation of Cosmic Rays: Statistical Study of Neutron Monitor Data Including Lomnický štít. Proceedings of 21 st ECRS-2003, Kosice, 9-12 September 2003, 1-5.

[45] Mailyan, M. and Chilingarian, A. (2010) Investigation of Diurnal Variations of Cosmic Ray Fluxes Measured with Using ASEC and NMDB Monitors. Advances in Space Research, 45, 1380-1387. https://doi.org/10.1016/j.asr.2010.01.027

[46] Kozyarivsky, V., Lidvansky, A.S., Petkov, V. and Tulupova, T. (2005) Mean Diurnal Variations of Cosmic Ray Intensity as Measured by the Baksan Surface and Underground Detectors. 29th ICRC Pune 2, Pune, 3-10 August 2005, 93-96.

[47] Kudela, K., Rybak, J., Antalova, A. and Storini, M. (2002) Time Evolution of Low-Frequency Periodicities in Cosmic Ray Intensity. Solar Physics, 205, 165-175. https://doi.org/10.1023/A:1013869322693

[48] Chowdhury, P. and Kudela, K. (2018) Quasi-Periodicities in Cosmic Rays and Time Lag with the Solar Activity at a Middle Latitude Neutron Monitor: 1982-2017. Astrophysics and Space Science, 363, 250. https://doi.org/10.1007/s10509-018-3467-y

[49] Chowdhury, P., Khan, M. and Ray, P. (2010) Evaluation of the Short and Intermediate Term Periodicities in Cosmic Ray Intensity during Solar Cycle 23. Planetary and Space Science, 58, 1045-1049. https://doi.org/10.1016/j.pss.2010.04.005

[50] El Borie, M. and Al Thoyaib, S. (2002) Power Spectrum of Cosmic-Ray Fluctuations during Consecutive Solar Minimum and Maximum Periods. Solar Physics, 209, 397-407. https://doi.org/10.1023/A:1021258430693

[51] Maghrabi, A., Makhmutov, V.S., Almutairi, M., Aldosari, A., et al. (2020) Cosmic Ray Observations by CARPET Detector Installed in Central Saudi Arabia-Preliminarily Results. Journal Solar Terrestrial Physics, 20, Article ID: 105194.

https://doi.org/10.1016/j.jastp.2020.105194 